\title{
Isobaric Peptide Termini Labeling
}

National Cancer Institute

\section{Source}

National Cancer Institute. Isobaric Peptide Termini Labeling. NCI Thesaurus. Code C161801.

A method for generating isobarically labeled peptides through the attachment of complementary isotope tags to each peptide termini. 